\title{
Hopf instantons, Chern-Simons vortices and Heisenberg ferromagnets
}

\author{
P. A. HORVÁTHY * \\ Laboratoire de Mathématiques et de Physique Théorique \\ Université de Tours \\ Parc de Grandmont, F-37200 TOURS (France)
}

\begin{abstract}
The dimensional reduction of the three-dimensional model (related to Hopf maps) of Adam et el. is shown to be equivalent to (i) either the static, fixed-chirality sector of the non-relativistic spinor-ChernSimons model in $2+1$ dimensions, (ii) or a particular Heisenberg ferromagnet in the plane.
\end{abstract}

\section{Scalar Chern-Simons vortices and Hopf instantons}

In the non-relativistic Chern-Simons model of Jackiw and Pi [1], one considers a scalar field $\Phi$ which satisfies a second-order non-linear Schrödinger equation,

$$
i D_{t} \Psi=\frac{D_{i} D^{i}}{2 m} \Psi-g|\Psi|^{2} \Psi=0,
$$

while the dynamics of the gauge field is governed by the Chern-Simons field/current identities. When the coupling constant $g$ is minus or plus the inverse of the Chern-Simons coupling constant $\kappa$, static solutions arise by solving instead the self-duality equations,

$$
D_{ \pm} \Psi \equiv\left(D_{1} \pm D_{2}\right) \Psi=0, \quad\left(D_{k}=\partial_{k}-i A_{k}\right),
$$

supplemented with one of the Chern-Simons equations, namely

$$
\kappa B \equiv \kappa \epsilon^{i j} \partial_{i} A^{j}=-\varrho,
$$

where $\varrho=\Phi^{*} \Phi$ is the particle density. Expressing the gauge potential from (1?2) one finds that the other Chern-Simons equations, $\kappa E^{i} \equiv-\kappa\left(\partial_{i} A^{0}+\partial_{t} A^{i}\right)=\epsilon^{i j} J^{j}$, merely fixes $A_{t}$. Then, inserting into (1.3) yields the Liouville equation, whose well-known solutions provide us with Chern-Simons vortices which carry electric and magnetic fields. The self-dual solutions represent furthermore the absolute minima of the energy, cf. [1].

In a recent paper, Adams, Muratori and Nash [2] consider instead a massless two-spinor $\Phi=\left(\begin{array}{l}\Phi_{+} \\ \Phi_{-}\end{array}\right)$ on ordinary 3-space, coupled to a (euclidean) Chern-Simons field. Their field equations read

$$
\begin{gathered}
D_{i} \sigma_{i} \Phi=0, \\
\Phi^{\dagger} \sigma_{i} \Phi=B_{i} .
\end{gathered}
$$

Note that this model only contains a (three-dimensional) magnetic but no electric field. These authors also mention that assuming independence of $x_{3}$ and setting $A_{3}=0$, their model will reduce to the planar self-dual Jackiw-Pi system, (1.2-3). The third component of (1.5) requires in fact

$$
\left|\Phi_{+}\right|^{2}-\left|\Phi_{-}\right|^{2}=B
$$

\footnotetext{
* e-mail: horvathy@univ-tours.fr
} 
the two other components imply, however, that either $\Phi_{+}$or $\Phi_{-}$has to vanish. Therefore, the reduced equations read finally one or the other of

$$
D_{ \pm} \Phi_{\mp}=0, \quad B= \pm\left|\Phi_{ \pm}\right|^{2}, \quad \text { and } \quad \Phi_{\mp}=0 .
$$

Fixing up the sign problem by including a Chern-Simons coupling constant $\kappa$, these equations look indeed formally the same as in the self-dual Jackiw-Pi case. They have, however, a slightly different interpretation: they are purely magnetic, while those of Jackiw and Pi have a non-vanishing electric field. Let us underline that the equations (1.7) differ from the second-order field equation (1.1).

\section{Spinor vortices}

Here we point out that the model of Adam et al. reduces rather more naturally to a particular case of our spinor model in $2+1$ dimensions [3]. In this theory, the 4-component Dirac spinor with components $\Phi_{-}, \chi_{-}, \chi_{+}$and $\Phi_{+}$satisfies the Lévy-Leblond equations [4]

$$
\left\{\begin{array}{c}
(\vec{\sigma} \cdot \vec{D}) \Phi+2 m \chi=0 \\
D_{t} \Phi+i(\vec{\sigma} \cdot \vec{D}) \chi=0
\end{array}\right.
$$

where $\Phi$ and $\chi$ are two-component 'Pauli' spinors $\Phi=\left(\begin{array}{l}\Phi_{-} \\ \Phi_{+}\end{array}\right)$and $\chi=\left(\begin{array}{l}\chi_{-} \\ \chi_{+}\end{array}\right)$. This non-relativistic Dirac-type equation is completed with the Chern-Simons equations

$$
\begin{aligned}
& B=(-1 / \kappa)\left(\left|\Phi_{+}\right|^{2}+\left|\Phi_{-}\right|^{2}\right), \\
& E_{i}=(1 / \kappa) \epsilon_{i j} J_{j}, \quad J_{j}=i\left(\Phi^{\dagger} \sigma_{j} \chi-\chi^{\dagger} \sigma_{j} \Phi\right) .
\end{aligned}
$$

In the static and purely magnetic case, $A_{t}=0$, and chosing $\chi_{+}=\chi_{-}=0$, the second equation in (2.2) is identically satisfied, leaving us with the coupled system

$$
\left\{\begin{array}{l}
D_{+} \Phi_{-}=0, \\
D_{-} \Phi_{+}=0, \\
B=(-1 / \kappa)\left(\left|\Phi_{+}\right|^{2}+\left|\Phi_{-}\right|^{2}\right) .
\end{array}\right.
$$

Choosing a fixed chirality, $\Phi_{-} \equiv 0$ or $\Phi_{+} \equiv 0$, yields furthermore either of the two systems

$$
\begin{aligned}
& D_{ \pm} \Phi_{\mp}=0, \\
& B=(-1 / \kappa)\left|\Phi_{ \pm}\right|^{2},
\end{aligned}
$$

which, for $\kappa=1$, are precisely (1.7). For both signs, the equations (2.4) reduce to the Liouville equation; regular solutions were obtained for $\Phi_{+}$when $\kappa<0$, and for $\Phi_{-}$when $\kappa>0$. They are again purely magnetic, and carry non-zero spin.

It would be easy keep both terms in (1.7) by allowing a non-vanishing (but still $x_{3}$-independent) $A_{3}$. Then one would loose the equations $D_{ \pm} \Phi_{\mp}=0$, however. The impossibility to having both components in (2.3) but not in (1.7) comes from the type of reduction performed: while for spinors one eliminates nonrelativistic time, (1.7) comes from a spacelike reduction. The difference is also related to the structure of the Lévy-Leblond equation (2.1), which can be obtained by lightlike reduction from a massless Dirac equation in 4-dimensions, while (1.4) comes by spaceloke reduction [3].

It is interesting to observe that eliminating $\chi$ in favor of $\Phi$ in the Lévy-Leblond equation (2.3) yields

$$
i D_{t} \Phi=\left[-\frac{1}{2 m} D_{i} D^{i}+\frac{1}{2 m \kappa}\left(\left|\Phi_{+}\right|^{2}+\left|\Phi_{-}\right|^{2}\right) \sigma_{3}\right] \Phi .
$$


For both chiralities, we get hence a second-order equation of the Jackiw-Pi form (1.1), but with opposite signs i.e., with attractive/repulsive coupling.

It is worth noting that minima of the energy correspond to the coupled equations (2.3) and not to (2.4). In fact, the identity

$$
|\vec{D} \Phi|^{2}=\left|D_{+} \Phi_{-}\right|^{2}+\left|D_{-} \Phi_{+}\right|^{2}-(1 / 2 m \kappa)|\Phi|^{2} \Phi^{\dagger} \sigma_{3} \Phi+\text { surface terms }
$$

shows that the energy of a field configuration,

$$
H=\int\left\{(1 / 2 m)|\vec{D} \Phi|^{2}+(1 / 2 m \kappa)|\Phi|^{2} \Phi^{\dagger} \sigma_{3} \Phi\right\} d^{2} \vec{x}
$$

is actually

$$
H=\frac{1}{2 m} \int d^{2} \vec{r}\left\{\left|D_{+} \Phi_{-}\right|^{2}+\left|D_{-} \Phi_{+}\right|^{2}\right\},
$$

which is positive definite, $H \geq 0$, provided the currents vanish at infinity. The "Bogomolny" bound is furthermore saturated precisely when (2.3) holds. Its solutions are therefore stable; (2.3) should be considered as the true self-duality condition.

\section{Heisenberg ferromagnets}

The relative minus sign of the component densities in the "provisional" formula (1.6) differs from ours in (2.3), and is rather that in the 2-dimensional Heisenberg model studied by Martina et al. [5]. Here the spin, represented by a unit vector $\mathbf{S}$, satisfies the Landau-Lifschitz equation $\partial_{t} \mathbf{S}=\mathbf{S} \times \triangle \mathbf{S}$. In the so-called tangent-space representation, $\mathbf{S}$ is replaced by two complex fields, $\Psi_{+}$and $\Psi_{-}$, each of which satisfies a (second-order) non-linear Schrödinger equation,

$$
i D_{t} \Psi_{ \pm}=-\left[D_{i} D^{i}+8\left|\Psi_{ \pm}\right|^{2}\right] \Psi_{ \pm}
$$

as well as a geometric constraint, $D_{+} \Psi_{-}=D_{-} \Psi_{+}$. The covariant derivatives here refer to a Chern-Simonstype abelian gauge field,

$$
\begin{aligned}
& B=-8\left(\left|\Psi_{+}\right|^{2}-\left|\Psi_{-}\right|^{2}\right), \\
& E_{i}=8 \epsilon_{i j} J_{j}, \quad J_{i}=\left(\Psi_{+}^{*} D_{i} \Psi_{+}-\Psi_{+}\left(D_{i} \Psi_{+}\right)^{*}\right)-\left(\Psi_{-}^{*} D_{i} \Psi_{-}-\Psi_{-}\left(D_{i} \Psi_{-}\right)^{*}\right) .
\end{aligned}
$$

It is now easy to check that in the static and purely magnetic case, these equations can be solved by the first-order coupled system

$$
\begin{aligned}
& D_{ \pm} \Psi_{\mp}=0 \\
& B=-8\left(\left|\Psi_{+}\right|^{2}-\left|\Psi_{-}\right|^{2}\right) .
\end{aligned}
$$

For $\Psi_{+}=0$ or $\Psi_{-}=0$, we get once again the equation of Adams et al.. In the general case, (3.3) leads to an interesting generalization of the Liouville equation : making use of its conformal properties, Martina et al. have shown that it can be transformed into the "sinh-Gordon" form

$$
\triangle \sigma=-\sinh \sigma
$$

where $\sigma$ is suitably defined from $\Psi_{+}$and $\Psi_{-}$. Although this equation has no finite-energy regular solution defined over the whole plane [6], it admits doubly-periodic solutions i. e. solutions defined in cells with 
periodic boundary conditions on the boundary [7]. This generalises the results of Olesen [8] in the scalar case. A similar calculation applied to the general SD equations, (2.3), of our spinor model would yield

$$
\triangle \sigma=-\cosh \sigma
$$

whose (doubly periodic) solutions could be interpreted as non-linear superpositions of the chiral vortices in [DHP].

\section{References}

[1] R. Jackiw and S-Y. Pi, Phys. Rev. Lett. 64, 2969 (1990); Phys. Rev. D42, 3500 (1990); For reviews, see, e. g., R. Jackiw and S-Y. Pi, Prog. Theor. Phys. Suppl. 107, 1 (1992) or G. Dunne, Self-Dual Chern-Simons solitons. Springer Lecture Notes in Physics (New Series) 36, (1995).

[2] C. Adam, B. Muratori, and C. Nash, Phys. Lett. B 479, 329 (2000).

[3] C. Duval, P. A. Horváthy and L. Palla, Phys. Rev. D52, 4700 (1995); Ann. Phys. (N.Y.) 249, 265 (1996). The same self-dual equations arise in the relativistic model of Y. M. Cho, J. W. Kim, and D. H. Park, Phys. Rev. D45, 3802 (1992).

[4] J-M. Lévy-Leblond, Comm. Math. Phys. 6, 286 (1967).

[5] L. Martina, O. K. Pashaev, G. Soliani, Phys. Rev. B48, 15787 (1993).

[6] G. Dunne, R. Jackiw, S.-Y. Pi, Trugenberger, Phys. Rev. D43, 1332 (1991).

[7] A. C. Ting, H. H. Chen and Y. C. Lee, Phys. Rev. Lett. 53, 1348 (1984); Physica 26D, 37 (1987).

[8] P. Olesen, Phys. Lett. B265, 361 (1991); ibid. B268, 389 (1991). 